\title{
ChemComm
}

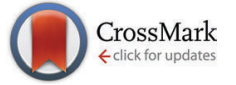

Cite this: Chem. Commun., 2015 51,6111

Received 27th January 2015,

Accepted 20th February 2015

DOI: $10.1039 / \mathrm{c} 5 \mathrm{cc00773a}$

www.rsc.org/chemcomm

\section{Aqueous phase nitric oxide detection by an amine-decorated metal-organic framework $\dagger$}

\author{
Aamod V. Desai, Partha Samanta, Biplab Manna and Sujit K. Ghosh*
}

\begin{abstract}
Selective and sensitive aqueous phase nitric oxide (NO) detection has been demonstrated by implementing an unsophisticated approach of ligand modulation in a porous, robust metal-organic framework (MOF). The detection is achieved through deamination by NO in an amine-decorated luminescent MOF. This is the first report of employing a physiologically stable, functionalized MOF as a NO sensor.
\end{abstract}

Nitric oxide (NO) has been widely recognized as a vital biological signalling agent and identified as a key neurotransmitter. ${ }^{1}$ It has also been found to be involved as a protective and regulatory agent in several physiological and pathological processes like cellular adhesion, renal functioning, leukocyte adhesion inhibition and bronchodilation. Despite reports of diverse functionality of NO in biological systems, ${ }^{2}$ the mechanisms of such activity are not completely understood. NO has high diffusivity and its short lived nature makes it a difficult molecule to investigate with respect to its reactivity pathways. ${ }^{3}$ To enhance our insightful understanding of in vivo NO functioning a number of techniques have been resorted to, including chemiluminescence, colorimetry, electron paramagnetic resonance and electrochemistry. ${ }^{4}$ Fluorescence-based methods score over others due to their higher sensitivity to analytes, non-invasive nature, quick response, ease of sample processing and simplicity in real-time monitoring when coupled with microscopic imaging. ${ }^{5}$ To exploit these advantages, artificial fluorescent probes targeting such biologically relevant species and incorporating all the attributes are desired.

The conventional fluorometric strategies for NO recognition by synthetic molecules have focused on ortho-diamine probes and metal-based complexes. The diamine appended systems have relied on NO activated triazole formation to disengage the photoinduced electron transfer (PET) process, ${ }^{6}$ while those based on metal-centred complexes have utilized the reductive

Indian Institute of Science Education and Research (IISER), Pune, Dr. Homi Bhabha Road, Pashan, Pune, 411008, India. E-mail: sghosh@iiserpune.ac.in; Web: http:// www.iiserpune.ac.in/ sghosh; Fax: +9120 2589 8022; Tel: +91 2025908076 $\dagger$ Electronic supplementary information (ESI) available: Synthesis details, PXRD patterns, ${ }^{1} \mathrm{H}$-NMR, FT-IR spectra, solid-state reflectance spectra, gas adsorption profiles and photophysical studies of the MOF. See DOI: 10.1039/c5cc00773a nature of NO towards the multivariate oxidation states of metals. ${ }^{7}$ Despite considerable progress in the development of in vivo targeted synthetic probes, there exist a few intrinsic constraints. The poor water solubility of organic reporter molecules hinders their applicability and the limited compatibility of inorganic complexes with endogenic conditions limits their utility. In pursuit of overcoming these shortcomings, development of relevant newer materials and/or improved synthetic modulations is an active aspect of current research in this domain.

Metal-organic frameworks (MOFs) have emerged as an exciting class of porous materials, which are constructed from organic linkers and inorganic cationic nodes. ${ }^{8}$ The possibility of tuning the pore surface actuates the fabrication of a host system sought for selective molecular recognition. The porous nature improves the extent of host-guest interactions by virtue of pre-concentration and serves to examine the confinement effect on the target molecule. ${ }^{9}$ The ordered accessible channel enhances molecular diffusivity to hasten the response time. Owing to the chemical and thermal stability of such ordered networks, certain MOFs can be utilized in environments with diverse chemical and/or physical extremities. Inclusion of all these attributes has made MOFs as a potential candidate for sensing and biomedical applications. ${ }^{10}$ The feasible accessory of luminescence as a signal transduction mechanism affords a notable subordinate classification of luminescent metal-organic frameworks (LMOFs). ${ }^{11}$ LMOFs furnish these materials as probes to selectively recognize a variety of molecules and ions by a detectable luminescence response.

MOFs have found utility as NO storage and delivery agents by rational application of the porous character of these materials. ${ }^{12}$ Yet the extension of the commonly applied approaches for NO detection to MOF-based sensors has been rare. The only known report has employed the reduction of the $\mathrm{Cu}^{2+}$ metal centre by NO as a function of fluorescence change. ${ }^{13}$ Although the mechanism in play affords the formation of $\mathrm{Cu}(\mathrm{I})$ metal centres, MOFs based on $\mathrm{Cu}(\mathrm{I})$ and carboxylate anions are not known to be stable in the aqueous phase and fluorescence response may arise from the disintegration of the ordered framework in such cases. It is therefore important to develop MOF systems which 
can be stable in different solvent environments. The superior approach to address the objective would be to append the appropriate functionality to the organic ligand. This could avoid the concerns regarding the stability of the probe system and also improve specificity to analyte response.

Along these lines we sought to encompass feasible approaches into LMOFs by applying functional organic moieties for constructing such porous networked probes. Recently the potency of a free primary aromatic mono-amine and an ortho-hydroxyamino group as a NO sensory functional group under aerobic conditions by a deamination mechanism has been reported. ${ }^{14}$ The active moiety was tagged to a fluorophore and the alteration of the photoinduced electron transfer (PET) process corresponded to the deamination reaction. Enthused with this knowledge, we sought to incorporate the free mono-amine aromatic group into a small ligand of a chemically stable, luminescent and porous MOF viz. UiO-66@ $\mathrm{NH}_{2}\left(\mathbf{1}-\mathrm{NH}_{2}\right),{ }^{15}$ for detection of NO (Scheme 1). In contrast to the reaction of NO with aromatic amines under inert conditions, ${ }^{16}$ the present case involves deamination in a MOF as the reaction proceeds in an aerated aqueous medium. The MOF is stable under physiological conditions for several hours and constitutes relatively non-toxic metal centres and bears free primary amine moieties as pendant groups in the porous channels (Fig. 1). The origin of fluorescence in this MOF is primarily from the organic ligand and thus seemingly the modulation of the active site as a consequence of the deamination reaction can be monitored in the fluorescence response directly without the interference from any background metal-based emission.

To examine the response of $\mathbf{1}-\mathbf{N H}_{\mathbf{2}}$ towards NO, the desolvated phase of the MOF (dispersed in HEPES buffer $10 \mathrm{mM}, \mathrm{pH} 7.4$ ) was excited at $325 \mathrm{~nm}$ and the emission profile was recorded between 340 and $630 \mathrm{~nm}$ followed by NO addition. A smooth emission curve peaking at $432 \mathrm{~nm}$ was observed, which underwent a significant change upon treatment with NO (Fig. 2). Notably the fluorescence change is more than $80 \%$ upon addition of only 1.0 equivalent of NO. To ascertain the structural integrity of the probe compound and corroborate with the understood mechanism, powder X-ray diffraction (PXRD), ${ }^{1} \mathrm{H}-\mathrm{NMR}$ and Fourier-transform infrared (FT-IR) spectroscopy, gas adsorption, scanning electron microscopy (SEM) and ultraviolet reflectance experiments were performed before and after NO addition. The PXRD patterns confirmed the stability of the probe towards the target species under physiological conditions (Fig. S1, ESI $\dagger$ ). SEM images also

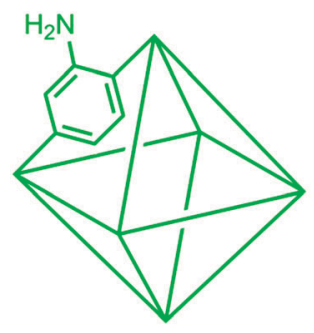

Fluorescent

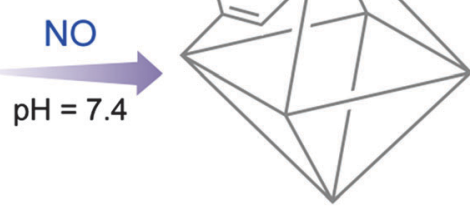

Non-Fluorescent
Scheme 1 Schematic illustration of nitric oxide detection by a functionalized MOF.

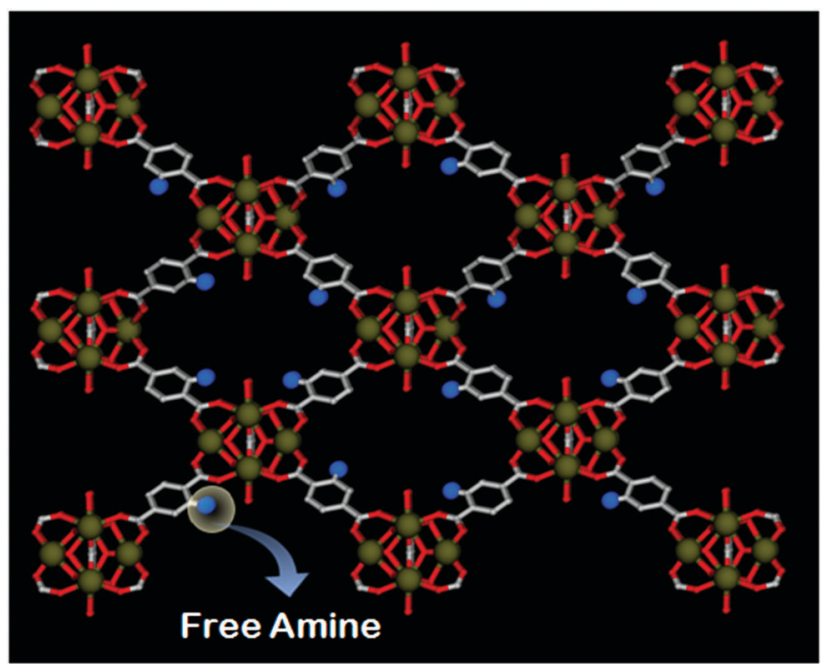

Fig. 1 Porous channels bearing free primary amine in 1- $\mathbf{N H}_{\mathbf{2}}$ (colour - C: grey, $\mathrm{N}$ : blue, O: red, and Zr: dark yellow; hydrogen atoms are omitted for clarity).

confirm the morphological stability of the probe after NO treatment (Fig. S19, ESI $\dagger$ ). ${ }^{1} \mathrm{H}-\mathrm{NMR}$ spectra of the digested samples of 1-NH $\mathbf{N H}_{2}$ before and after NO treated phases (Fig. S3, ESI $\dagger$ ) along with the decrement of the $\mathrm{N}-\mathrm{H}$ bending peak at $1650 \mathrm{~cm}^{-1}$ in the FT-IR spectrum (Fig. S2, ESI $\dagger$ ) and the $\sim 10 \mathrm{~nm}$ shift in the reflectance spectrum are in accord with the deamination mechanism (Fig. S5, ESI $\dagger$ ). Low temperature $\mathrm{N}_{2}$ and $\mathrm{CO}_{2}$ gas adsorption measurements were performed on both the compounds. As anticipated, the $\mathrm{N}_{2}$ adsorption $(77 \mathrm{~K})$ uptake amount subtly enhances in the case of the NO-treated compound (Fig. S17, $\mathrm{ESI} \dagger)$. The $\mathrm{CO}_{2}(195 \mathrm{~K})$ uptake amount in the case of the NO treated phase appreciably reduces from that of the parent compound (Fig. S18, ESI $\dagger$ ), which substantiates the correlation of lessening of active amine sites with the deamination reaction, since the effect of the amino group on $\mathrm{CO}_{2}$ adsorption in MOFs is well understood. ${ }^{17}$

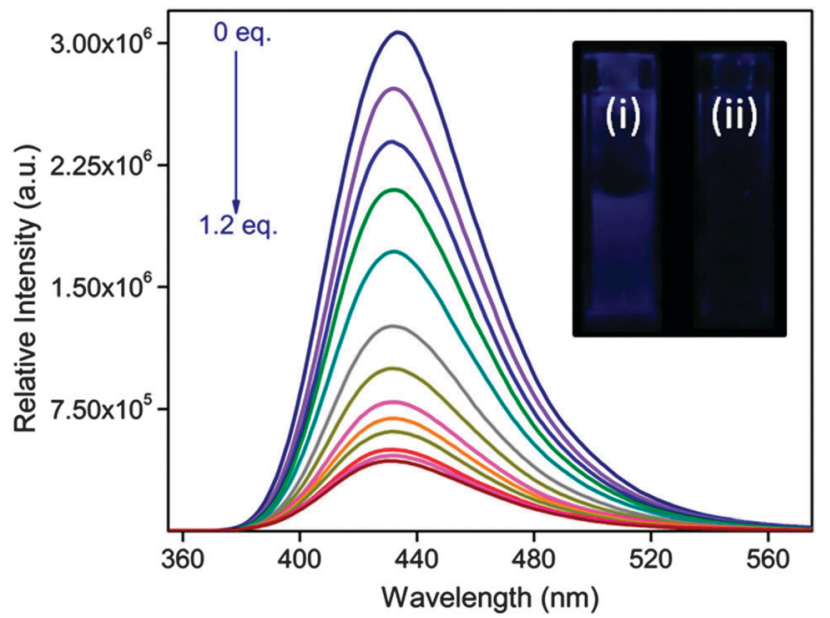

Fig. 2 Change in fluorescence intensity of 1- $\mathrm{NH}_{2}$ upon incremental addition of $0.1 \mathrm{mM}$ NO solution. Inset - fluorescence of 1-NH $\mathrm{NH}_{2}$ (i) before and (ii) after $\mathrm{NO}$ addition. 
The fluorescence response to incremental treatment of NO was recorded and gradual quenching of the MOF fluorescence could be observed (Fig. 2). The fluorescence change at a low concentration gives an excellent linear fitting $\left(R^{2}=0.9997\right)$ with a quenching constant of $4.15 \times 10^{5} \mathrm{M}^{-1}$ (Fig. S6, ESI $\dagger$ ). The high quenching constant value emphasizes the strong interactions between the highly diffusive NO and the MOF bearing pendant free amine. The LOD (limit of detection) for the probe was calculated to be $0.575 \mu \mathrm{M}$ (Fig. S20, Tables S1 and S2, ESI $\dagger$ ) which is close to the relevant cellular range of NO. ${ }^{18}$ Additionally the response of NO towards an analogous MOF bearing no free functionality viz. UiO-66 (2) was checked under similar conditions (Fig. S16, ESI $\dagger$ ). The fluorescence response was not perturbed considerably, which highlights the role of the primary amine group in the sensing process. Likewise, the response of NO towards the ligand only was recorded. The fluorescence response is not altered at such a concentration, which clearly emphasizes the superiority of an ordered framework for the present application. These results also suggest that LMCT-based fluorescence dominates in the case of $\mathbf{1}-\mathbf{N H}_{2}$, which renders low concentration detection. ${ }^{19}$

Exclusivity in detection is imperative for a real-time sensor system. To validate this aspect, the responses of $\mathbf{1}-\mathbf{N H}_{\mathbf{2}}$ towards different possible competitive RNS and ROS species (2 equiv.) like $\mathrm{H}_{2} \mathrm{O}_{2}, \mathrm{ClO}^{-}$, ${ }^{\bullet} \mathrm{OH},{ }^{1} \mathrm{O}_{2}, \mathrm{NO}_{3}{ }^{-}$and $\mathrm{NO}_{2}{ }^{-}$were recorded. In a typical experiment the particular RNS/ROS species were added to the solution of 1- $\mathbf{N H}_{2}$ in HEPES and allowed to equilibrate for 5 minutes. No substantial change was observed for any of the species (Fig. 3a), affirming the selective performance of the probe.

Encouraged by these results we sought to check the consistency of the performance in the concurrent presence of the other RNS/ROS species. In this experiment, the particular RNS/ ROS species was added to the solution containing $\mathbf{1}-\mathbf{N H}_{\mathbf{2}}$ and equilibrated for 5 min followed by recording of the emission spectrum. To this solution NO was added and the response was monitored after equilibration. As anticipated, the presence of other RNS/ROS species does not alter the activity of NO towards the functionalized MOF compound (Fig. 3b). From the perspective of applicability in complex biological systems, these results render the present MOF relevant for NO detection, avoiding offtarget reactivity and false response.

In summary, we have presented a novel approach of regulating the ligand functionality in a MOF as a function to probe the presence of NO in an aqueous environment. Hitherto only metal-based emission in MOFs has been demonstrated as a tool to detect NO, but the aspect of a functional pore surface seeks to be studied for better applicability. Deamination by NO in an amine-decorated MOF has been tapped as a process to monitor its presence. The MOF performs the sensory activity in a selective manner, even in the presence of potentially interfering RNS/ROS species. Although few organic compounds are known as NO sensors via a deamination pathway, this report presents a new platform of stable MOFs for investigation of such reaction-based sensors. Owing to accessible and ordered porosity, MOFs present a uniquely superior of class of sensors with enhanced host-guest interactions and tuneable functionalization of building blocks can render their use as real-time
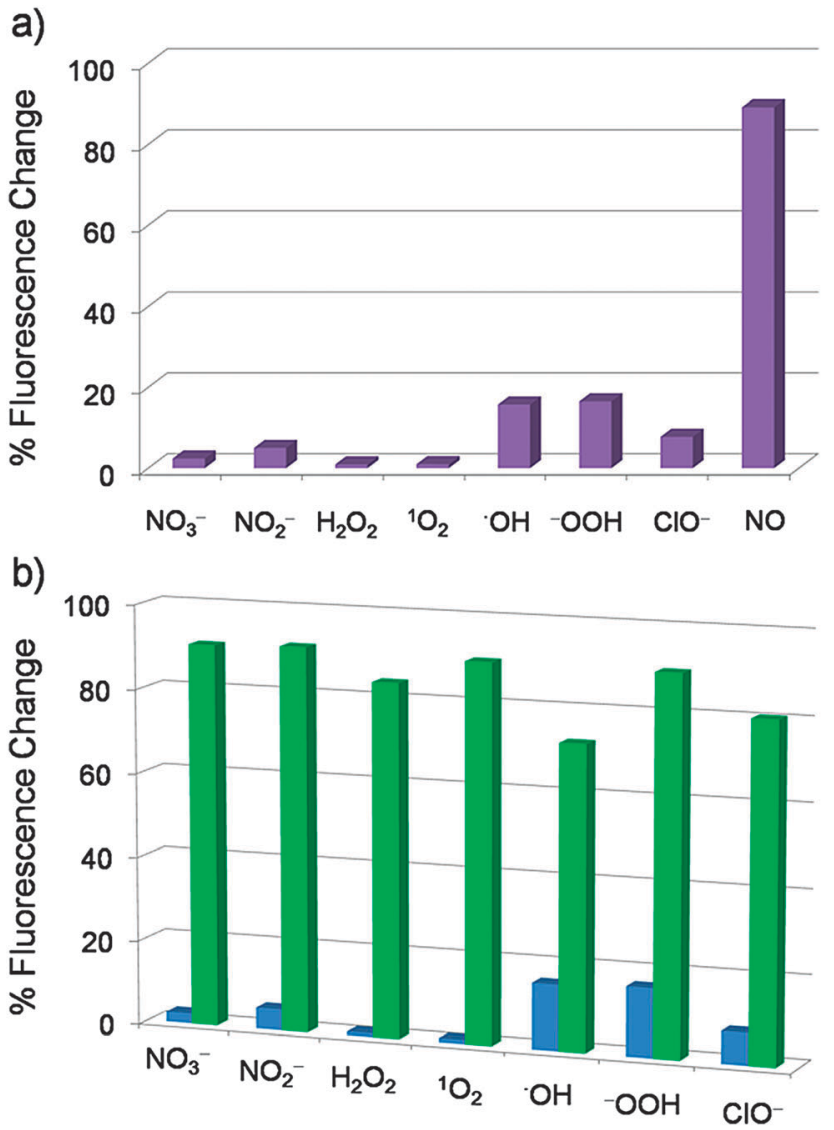

Fig. 3 (a) The fluorescence change of $\mathbf{1}-\mathbf{N H}_{\mathbf{2}}$ towards various RNS/ROS analytes. (b) The fluorescence change of $\mathbf{1}-\mathbf{N H}_{\mathbf{2}}$ in the presence of the particular RNS/ROS species (blue) followed by NO addition (green).

sensors for a variety of applications. We believe that the present work will stimulate the research in the field of MOF-based sensors for NO detection and will propel the goal of attaining probes for relevant biological applications.

A.V.D. and P.S. contributed equally to this work. P.S. and B.M. are thankful to UGC and CSIR, respectively, for research fellowships. We are grateful to IISER, Pune, for research facilities. DST (Project No. GAP/DST/CHE-12-0083) is acknowledged for the financial support.

\section{Notes and references}

1 (a) J. Garthwaite, S. L. Charles and R. Chess-Williams, Nature, 1988, 336, 385; (b) V. Calabrese, C. Mancuso, M. Calvani, E. Rizzarelli, D. A. Butterfield and A. M. G. Stella, Nat. Rev. Neurosci., 2007, 8, 766.

2 (a) C. Bogdan, Nat. Immunol., 2001, 2, 907; (b) B. G. Hill, B. P. Dranka, S. M. Bailey, J. R. Lancaster Jr and V. M. Darley-Usmar, J. Biol. Chem., 2010, 285, 19699; (c) P. G. Wang, M. Xian, X. Tang, X. Wu, Z. Wen, T. Cai and A. J. Janczuk, Chem. Rev., 2002, 102, 1091.

3 (a) J. R. Lancaster Jr, Nitric Oxide, 1997, 1, 18; (b) D. D. Thomas, X. Liu, S. P. Kantrow and J. R. Lancaster Jr, Proc. Natl. Acad. Sci. U. S. A., 2001, 98, 355.

4 (a) T. Nagano and T. Yoshimura, Chem. Rev., 2002, 102, 1235; (b) P. N. Coneski and M. H. Schoenfisch, Chem. Soc. Rev., 2012, 41, 3753.

5 A. Minta, J. P. Y. Kao and R. Y. Tsien, J. Biol. Chem., 1989, 264, 8171.

6 (a) E. Sasaki, H. Kojima, H. Nishimatsu, Y. Urano, K. Kikuchi, Y. Hirata and T. Nagano, J. Am. Chem. Soc., 2005, 127, 3684; (b) H. Yu, Y. Xiao and L. Jin, J. Am. Chem. Soc., 2012, 134, 17486; 
(c) Y. Yang, S. K. Seidlits, M. M. Adams, V. M. Lynch, C. E. Schmidt, E. V. Anslyn and J. B. Shear, J. Am. Chem. Soc., 2010, 132, 13114; (d) Y.-Q. Sun, J. Liu, H. Zhang, Y. Huo, X. Lv, Y. Shi and W. Guo, J. Am. Chem. Soc., 2014, 136, 12520; (e) M. J. Marn, P. Thomas, V. Fabregat, S. V. Luis, D. A. Russell and F. Galindo, ChemBioChem, 2011, 12, 2471.

7 (a) M. H. Lim and S. J. Lippard, Acc. Chem. Res., 2007, 40, 41; (b) M. H. Lim, D. Xu and S. J. Lippard, Nat. Chem. Biol., 2006, 2, 375; (c) X. Hu, J. Wang, X. Zhu, D. Dong, X. Zhang, S. Wu and C. Duan, Chem. Commun., 2011, 47, 11507; (d) Y. Chen, W. Guo, Z. Ye, G. Wang and J. Yuan, Chem. Commun., 2011, 47, 6266.

8 (a) S. S. Nagarkar, A. V. Desai and S. K. Ghosh, Chem. - Asian J., 2014, 9, 2358; (b) S. Kitagawa, R. Kitaura and S. Noro, Angew. Chem., Int. Ed., 2004, 43, 2334; (c) M. Kim and S. M. Cohen, CrystEngComm, 2012, 14, 4096; (d) H.-C. Zhou, J. R. Long and O. M. Yaghi, Chem. Rev., 2012, 112, 673; (e) M. C. Das and P. K. Bharadwaj, J. Am. Chem. Soc., 2009, 131, 10942; $(f)$ W. Lu, Z. Wei, Z.-Y. Gu, T.-F. Liu, J. Park, J. Park, J. Tian, M. Zhang, Q. Zhang, T. Gentle III, M. Boscha and H.-C. Zhou, Chem. Soc. Rev., 2014, 43, 5561; $(g)$ S. Sen, N. N. Nair, T. Yamada, H. Kitagawa and P. K. Bharadwaj, J. Am. Chem. Soc., 2012, 134, 19432.

9 R. Xiong, K. Odbadrakh, A. Michalkova, J. P. Luna, T. Petrova, D. J. Keffer, D. M. Nicholson, M. A. Fuentes-Cabrera, J. P. Lewis and J. Leszczynski, Sens. Actuators, B, 2010, 148, 459.

10 (a) P. Horcajada, R. Gref, T. Baati, P. K. Allan, G. Maurin, P. Couvreur, G. Férey, R. E. Morris and C. Serre, Chem. Rev., 2012, 112, 1232; (b) A. C. McKinlay, R. E. Morris, P. Horcajada, G. Férey, R. Gref, P. Couvreur and C. Serre, Angew. Chem., Int. Ed., 2010, 49, 6260; (c) J. D. Rocca, D. Liu and W. Lin, Acc. Chem. Res., 2011, 44, 957; (d) S. S. Nagarkar, T. Saha, A. V. Desai, P. Talukdar and S. K. Ghosh, Sci. Rep., 2014, 4, DOI: 10.1038/srep07053; (e) Y. Ikezoe, G. Washino, T. Uemura, S. Kitagawa and H. Matsui, Nat. Mater., 2012, 11, 1081.

11 (a) M. D. Allendorf, C. A. Bauer, R. K. Bhakta and R. J. T. Houk, Chem. Soc. Rev., 2009, 38, 1330; (b) Y. Cui, Y. Yue, G. Qian and
B. Chen, Chem. Rev., 2012, 112, 1126; (c) L. E. Kreno, K. Leong, O. K. Farha, M. Allendorf, R. P. Van Duyne and J. T. Hupp, Chem. Rev., 2012, 112, 1105.

12 (a) S. Shimomura, M. Higuchi, R. Matsuda, K. Yoneda, Y. Hijikata, Y. Kubota, Y. Mita, J. Kim, M. Takata and S. Kitagawa, Nat. Chem., 2010, 2, 633; (b) B. Xiao, P. J. Byrne, P. S. Wheatley, D. S. Wragg, X. Zhao, A. J. Fletcher, K. M. Thomas, L. Peters, J. S. O. Evans, J. E. Warren, W. Zhou and R. E. Morris, Nat. Chem., 2009, 1, 289; (c) M. I. H. Mohideen, B. Xiao, P. S. Wheatley, A. C. McKinlay, Y. Li, A. M. Z. Slawin, D. W. Aldous, N. F. Cessford, T. Duren, X. Zhao, R. Gill, K. M. Thomas, J. M. Griffi n, S. E. Ashbrook and R. E. Morris, Nat. Chem., 2011, 3, 304; (d) R. E. Morris and P. S. Wheatley, Angew. Chem., Int. Ed., 2008, 47, 4966.

13 P. Wu, J. Wang, C. He, X. Zhang, Y. Wang, T. Liu and C. Duan, Adv. Funct. Mater., 2012, 22, 1698.

14 (a) A. Beltrán, M. I. Burguete, D. R. Abánades, D. Prez-Sala, S. V. Luis and F. Galindo, Chem. Commun., 2014, 50, 3579; (b) T. Itoh, K. Nagata, Y. Matsuya, M. Miyazaki and A. Ohsawa, J. Org. Chem., 1997, 62, 3582; (c) T.-W. Shiue, Y.-H. Chen, C.-M. Wu, G. Singh, H.-Y. Chen, C.-H. Hung, W.-F. Liaw and Y.-M. Wang, Inorg. Chem., 2012, 51, 5400.

15 S. J. Garibay and S. M. Cohen, Chem. Commun., 2010, 46, 7700.

16 (a) J. G. Nguyen, K. K. Tanabe and S. M. Cohen, CrystEngComm, 2010, 12, 2335; (b) M. J. Ingleson, R. Heck, J. A. Gould and M. J. Rosseinsky, Inorg. Chem., 2009, 48, 9986.

17 (a) R. Vaidhyanathan, S. S. Iremonger, G. K. H. Shimizu, P. G. Boyd, S. Alavi and T. K. Woo, Science, 2010, 330, 650; (b) R. Vaidhyanathan, S. S. Iremonger, G. K. H. Shimizu, P. G. Boyd, S. Alavi and T. K. Woo, Angew. Chem., Int. Ed., 2012, 51, 1826; (c) C. Montoro, E. García, S. Calero, M. A. Pérez-Fernández, A. L. López, E. Barea and J. A. R. Navarro, J. Mater. Chem., 2012, 22, 10155.

18 B. Joarder, A. V. Desai, P. Samanta, S. Mukherjee and S. K. Ghosh, Chem. - Eur. J., 2015, 21, 965.

19 Z. Hu, B. J. Deibert and J. Li, Chem. Soc. Rev., 2014, 43, 5815. 